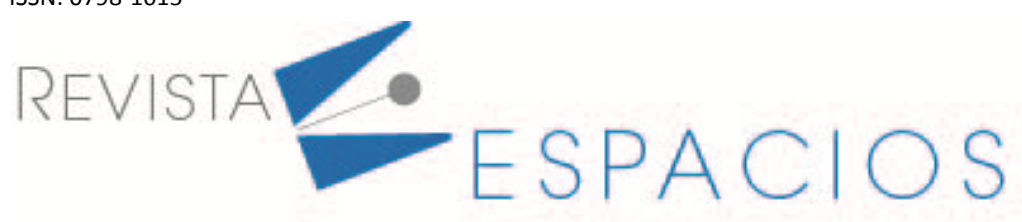

\title{
El problema de la formación de los valores patrióticos en el proceso educativo de la escuela superior
}

\section{The problem of patriotic values formation in the higher school educational process}

\author{
LARINA, Tatiana V. ${ }^{1}$ \\ SUKHANOVA, Anastasiia S. ${ }^{2}$ \\ KODIROV, Bakhtiyor R. ${ }^{3}$ \\ KUCHINSKAYA, Elizaveta A. ${ }^{4}$ \\ BORISOVA, Elena . $^{5}$
}

\begin{abstract}
Resumen
El objetivo del articulo es presentar un análisis de los aspectos prácticos de la formación de valores patrióticos por medio de una lengua extranjera en el proceso educativo de la escuela superior. A base del análisis de diferentes estudios en el campo de la formación de valores, fue elaborada una estructura clara de los materiales didácticos. El trabajo experimental ha demostrado que el medio eficaz para la formación de valores patrióticos es un conjunto de materiales didácticos con las tareas de orientación patriótica.
\end{abstract}

Palabras clave: valores patrióticos, escuela superior, proceso educativo, estudiantes

\begin{abstract}
The purpose of the article is to present an analysis of the practical aspects of the patriotic values formation by means of a foreign language in the higher school educational process. Based on the studies of values formation process, a clear structure of the didactic materials has been elaborated. The experimental research has shown the effectiveness of the suggested didactic materials for the formation of patriotic values at the tertiary education level.
\end{abstract}

Key words: patriotic values, higher school, educational process, students

\section{Introducción}

El futuro de un país depende en gran medida de los jóvenes, quienes, con potencial, resolverán los problemas que enfrenta nuestro país. Como condición indispensable para la realización de los jóvenes en diversas áreas es su lealtad a la Patria, su disposición a trabajar por el bien del país, para protegerlo si es necesario (Adaeva, 2014, p. 4).

\footnotetext{
${ }^{1}$ El Centro Militar Educativo y Cientifico de las Fuerzas Aéreas de la "Academia del prof. N. Ye. Zhukovsky y Yu. A. Gagarin", Vorónezh, Rusia. tanialar2008@yandex.ru

2 El Centro Militar Educativo y Cientifico de las Fuerzas Aéreas de la "Academia del prof. N. Ye. Zhukovsky y Yu. A. Gagarin", Vorónezh, Rusia. suhnastasia@mail.ru

${ }^{3}$ Institución Educativa Presupuestaria Federal de Educación Profesional Superior "Universidad Técnica Estatal de Voronezh". bakhtiyor_0663@mail.ru

${ }^{4}$ Academia de Defensa Aérea de las Fuerzas Armadas de la Federación de Rusia. kuchinskaya@list.ru

${ }^{5}$ Institución Estatal "El Instituto de Investigación del Servicio Federal Penitenciario de Rusia". elenborisov@mail.ru
} 
Para esto, es importante crear condiciones que contribuyan a la formación de valores patrióticos (VP) de la generación más joven. El problema de la formación de valores de los estudiantes es uno de los más importantes para la educación superior moderna. La necesidad de poder actuar de acuerdo con una posición claramente desarrollada sobre la base de los valores de la sociedad hace que su formación sea relevante para los estudiantes. Las demandas de la sociedad orientan el proceso educativo de la escuela superior hacia el enriquecimiento de valores del contenido de la educación.

El objetivo del presente estudio es, sobre la base del análisis teórico y el trabajo experimental, analizar y considerar la formación de VP mediante la disciplina de "Lengua extranjera" y presentar un análisis de los aspectos prácticos de la formación de valores.

La hipótesis del estudio se basa en el hecho de que la formación de VP de los estudiantes que adquieren conocimientos en las universidades en el proceso de aprendizaje de una lengua extranjera (LE) será efectiva si se realiza con el potencial formativo y patriótico de la disciplina de "Lengua extranjera" sobre la base del uso de un complejo de materiales didácticos (MD).

Por consiguiente, era necesario examinar con más detalle el concepto de "valor". Estamos de acuerdo con la opinión del profesor A.M. Novikov, quien señala que los valores tienen un significado positivo, pueden satisfacer las necesidades de la persona, la sociedad, servir a sus intereses y objetivos (Novikov, 2013, p. 255). S.L. Rubinstein creía que los valores "se derivan de la relación entre el mundo y el hombre", incluyen componentes importantes para el individuo, lo que él creó "en el proceso de la historia es significativo para el hombre" (Rubinstein, 2003, p. 383).

El problema de la formación de valores, a saber, el VP, nos apunta a considerar la importancia del patriotismo. Por supuesto, el patriotismo es de gran importancia para cualquier estado. Los investigadores señalan que "la idea del patriotismo es inherente a cada país" (Shaidullin, Larina, 2016), el patriotismo es un núcleo ideológico sin el cual "ni el estado, ni la sociedad, ni las personas pueden existir" (Orlov, Lesnyak, 2006, p. 80). El patriotismo es uno de los valores más significativos propios a todas las esferas de la vida social (Bimakhanov et.al., 2018). A.N. Vyrshchikov y M.B. Kusmartsev llamaron a la alta idea patriótica como un marco en el que solo la espiritualidad de la sociedad rusa moderna y su futuro pueden construirse (Vyrshchikov, Kusmartsev, 2006, p. 6).

Para aclarar el concepto de VP, es necesario referirse a su interpretación en la literatura pedagógica. Para algunos investigadores, el VP es "ideas estables y positivamente significativas sobre los intereses nacionales de Rusia, determinadas por un conjunto de intereses equilibrados de la persona, la sociedad y el estado" (Salikov, 2011, p. 7). A su vez, los VP son vistos como "una educación espiritual muy compleja que incluye un sistema estrechamente interconectado de elementos civiles, nacionales e internacionales" (Rusetskiy, Gashchenko, 2000, p. 14). En su estudio, L.A. Gaschenko se basa en el hecho de que el VP es "una matriz sociocultural significativa, a través de la cual se evalúan los principales impulsores cívico - patrióticos de la personalidad, el entorno patriótico, el espacio etnopolítico en general" (Gashchenko, 2004, p. 11).

Por lo tanto, las tareas del proceso de formación de la VP son: profundización del conocimiento, preservación de la memoria y respeto por el pasado del país. La formación de la personalidad holística del alumno; implementación de la dirección de valor de la disciplina "Lengua extranjera".

A pesar del valor científico de la investigación disponible hasta la fecha, existe la necesidad de justificar el proceso de formación de VP en las universidades que estudian LE.

El estudio se basa en los aspectos principales del enfoque axiológico: A.A. Derkach (2004), A.V. Kiryakova (1996), V.A. Slastenin (2003); desarrollos científicos en el marco de un enfoque orientado a la personalidad: E.F. Zeer 
(2006), V.V. Serikov (1998), I.S. Yakimanskaya (1995); estudios sobre el enfoque sistémico: V.P. Bespalko (1977), I.V. Blauberg (1997).

El potencial educativo de la disciplina "Lengua extranjera" se revela en el trabajo de A.V. Konysheva (2017), en el estudio de S.S. Koroleva (2010). En estos se aclara el papel de LE en la formación de orientaciones de valor, el papel de LE en la educación moral y la educación del amor por la Patria enfatiza G.V. Rogova (2000).

En este sentido, es aconsejable considerar las disciplinas para tener la capacidad de generar valores. El análisis de la literatura científica ha demostrado que la disciplina "Lengua extranjera" tiene oportunidades significativas para la formación de valores durante el período de estudio en una Institución de educación superior (Konysheva, 2017). Dicha disciplina implica el estudio de la cultura y los valores de otros países, pero los valores asociados con su país también pueden formarse con éxito. Por lo tanto, el estudio de la LE requiere inevitablemente una apelación a la conciencia de valor de los estudiantes (Koroleva, 2010, p. 10). Esta disciplina tiene una orientación comunicativa y contribuye a la formación de las cualidades morales del individuo (Filashova, 2015, p. 14). G.V. Rogova enfatiza el papel de LE en la educación moral y la educación del amor por la Patria (Rogova, 2000).

Esta publicación científica se basa en investigaciones anteriores y presenta un examen más detallado de este problema.

\section{Metodología}

El trabajo experimental se llevó a cabo en la Academia Estatal. En el experimento participaron 90 estudiantes de 10 y 20 años de estudios (los grupos de control y experimental).

Para realizar el estudio, se utilizaron los siguientes métodos complementarios y de condicionamiento:

- teórico (análisis de la literatura psicologo-pedagógica sobre el tema del estudio; síntesis y sistematización de la experiencia de formación de valores; análisis comparativo, síntesis, sistematización de datos experimentales);

- empírico (conversación, estudio de los resultados de la actividad, observación pedagógica del proceso de formación de valores en las clases de LE).

Estos métodos permitirán obtener datos precisos, ya que se seleccionan de acuerdo con el propósito del estudio. Para el análisis de la literatura psico-pedagógica sobre el tema del estudio, se utilizó el método de generalización y sistematización. El análisis del proceso de formación de valores se llevó a cabo sobre la base de métodos de conversación y observación pedagógica.

\section{Resultados y discusión}

Creemos que es conveniente organizar el proceso de formación de PC sobre la base del desarrollo e inclusión de MD en el curso de las clases de LE. Bajo MD, después de L.A. Burylova, entendemos "herramientas de aprendizaje adicionales al libro de texto que contribuyen a resolver problemas didácticos en el proceso de aprendizaje a través de la realización de trabajos prácticos con ellos" (Burylova, 2004, p. 9).

Con el fin de analizar el proceso de formación de VP y sobre la base de la inclusión de MD, nos centraremos en los aspectos propuestos por E.I. Vishnevsky (Vishnevsky, 1988, p. 26-28). Para trabajar con MD, destacamos los aspectos más significativos que, en opinión del autor, distinguen la disciplina de la LE de las demás.

Un aspecto significativo que incluye la información, incluida la naturaleza patriótica, que reciben los estudiantes en las clases; la posibilidad de utilizar los vínculos inter-educativos. 
Un aspecto comunicativo implementado en la clase, ya que el lenguaje se utiliza como medio para recibir y transmitir información.

El aspecto organizativo, que consiste en la gestión del profesor de la actividad educativa, la posibilidad de seleccionar las técnicas y formas de trabajo correspondientes a las tareas de la clase, dirigir y organizar las actividades de los estudiantes.

El aspecto psicologo-emocional se manifiesta en la creación de una atmósfera favorable en las clases, apelando a la conciencia de valor de los estudiantes que perciben la información y forman su actitud hacia ella. La presencia de una reacción emocional al tema de la discusión determina la orientación axiológica del proceso de aprendizaje de la LE.

Todos los aspectos anteriores deben tenerse en cuenta en la formación de VP por medio de LE. Estos se reflejan en la orientación patriótica de MD, incluida en el curso de las clases de LE. Sobre la base de lo anterior, en términos significativos, los MD incluyen textos orientados a los valores, que actualizan los valores a nivel cognitivo. El aspecto comunicativo se manifiesta al concentrar la atención de los estudiantes en los problemas patrióticos, revelando su importancia, ayudado por la organización de la inclusión en el análisis de situaciones de orientación patriótica. El aspecto psicológico-emocional se refleja en el atractivo de las emociones y la resolución de situaciones de elección de valor. Los alumnos se incorporan a la actividad reflexiva y evaluativa durante la discusión, lo que contribuye a la formación de valores y la comprensión de su importancia.

La preparación del MD, además, debe basarse en los requisitos formulados en el marco de un enfoque orientado a la persona (Yakimanskaya, 1995, p. 35-36). Sobre la base de estos requisitos, el material de estudio debe cumplir con los siguientes criterios:

- considerar, estructurar y ampliar la experiencia subjetiva de los estudiantes;

- estimular a los estudiantes para el desarrollo personal, la autoexpresión durante las actividades educativas;

- proporcionar a los estudiantes la oportunidad de elegir en la realización de tareas con el fin de utilizar las formas de trabajo más productivas;

- supervisar no sólo el resultado, sino también el proceso de aprendizaje.

Este material teórico sirvió como base para el desarrollo del complejo MD y la realización de un estudio experimental. El objetivo del experimento fue demostrar la efectividad del uso de MD para formar VP en los estudiantes durante el estudio de la LE. El trabajo experimental se llevó a cabo durante las etapas de constatación (EC) y formación (EF) del experimento. El diagnóstico de control durante la etapa de afirmación en los grupos de control (GC) y experimental (GE) permitió evaluar el conjunto de valores asociados con el patriotismo de acuerdo con los componentes de la VP (cognitivo, emocional, motivacional, reflexivo) y con los niveles de formación: básico, medio, superior (Sukhanova, 2020, p. 735). Se analizaron y sistematizaron los datos obtenidos, con resultados similares en GC y GE.

Además, durante la etapa formativa del experimento, se aplicó un complejo de MD en las clases de LE. Los datos de rediagnóstico se compararon con los resultados de la etapa de constatación. Los resultados del estudio se presentan en la Tabla 1 (para el grupo de control) y la Tabla 2 (para el grupo experimental). 
Tabla 1

Título del cuadro o tabla Resumen comparativo de la dinámica de los niveles de formación de los valores patrióticos (grupo de control), \%

\begin{tabular}{|c|c|c|c|c|}
\hline \multirow{2}{*}{ Componente } & Etapa & Nivel básico & Nivel medio & Nivel superior \\
\hline \multirow{2}{*}{ cognitivo } & EC & 15,2 & 34,7 & 50,1 \\
\cline { 2 - 5 } & EF & 13,2 & 29,3 & 57,5 \\
\hline \multirow{2}{*}{ emocional } & EC & 19,6 & 34,7 & 36,3 \\
\cline { 2 - 5 } & EF & 7,8 & 45,7 & 55,9 \\
\hline \multirow{2}{*}{ motivacional } & EC & 32,6 & 28,3 & 39,1 \\
\cline { 2 - 5 } & EF & 26,8 & 26,3 & 46,9 \\
\hline \multirow{2}{*}{ reflexivo } & EC & 21,7 & 32,6 & 34,3 \\
\cline { 2 - 5 } & EF & 14,1 & 45,7 & 51,6 \\
\hline
\end{tabular}

Fuente: Elaboración propia (2020)

Tabla 2

Título del cuadro o tabla Resumen comparativo de la dinámica de los niveles de formación de los valores patrióticos (grupo experimental), \%

\begin{tabular}{|c|c|c|c|c|}
\hline \multirow{2}{*}{ Componente } & Etapa & Nivel básico & Nivel medio & Nivel superior \\
\hline \multirow{2}{*}{ cognitivo } & EC & 12,1 & 37,2 & 50,7 \\
\cline { 2 - 5 } & EF & 6,8 & 22,7 & 40,5 \\
\hline \multirow{2}{*}{ emocional } & EC & 20,3 & 33,7 & 46 \\
\cline { 2 - 5 } & EF & 9,1 & 25 & 32,3 \\
\hline \multirow{2}{*}{ motivacional } & EC & 40,6 & 27,1 & 56,9 \\
\cline { 2 - 5 } & EF & 6,8 & 36,3 & 43,5 \\
\cline { 2 - 5 } & EC & 19,9 & 36,6 & 63,6 \\
\hline
\end{tabular}

Fuente: Elaboración propia (2020)

En relación con el aspecto cognitivo, más de 1/3 de los encuestados mostraron un resultado medio o bajo, sin mostrarse con pleno conocimiento de la Patria y de su historia. Después del trabajo experimental, este indicador en el GE aumentó (70,5 \%), y en el GC se mantuvo en el mismo nivel.

En cuanto a la percepción emocional de la VP, también hay que destacar el aumento de la tasa en el GE (del $46 \%$ al 65,9\% para el nivel superior).

El análisis de las respuestas de los sujetos sobre la presencia de aspiraciones patrióticas y motivos de personalidad mostró que la mitad de los encuestados no mostró una posición clara en el deseo de asumir la responsabilidad del futuro del país. Sin embargo, este indicador aumentó un 24,6 \% en el GE (del 32,3 \% al $56,9 \%)$.

Con respecto a la autoevaluación de los estudiantes de la presencia de sus rasgos patrióticos inherentes, cabe señalar que $19,9 \%$ en GE y $21,7 \%$ en GC no se consideran dueños de las cualidades de un patriota. Después del trabajo experimental, esta tasa en GE aumentó a 63,6\%.

Por lo tanto, los datos experimentales revelaron tasas similares en GE y GC en promedio en todos los artículos. Después de las clases con el uso del complejo MD en el GE, todos los indicadores aumentaron. 
El análisis del material teórico permitió estructurar el MD en bloques de información (BI) e incluirlos en el curso de la lección. Cada BI tiene la misma estructura y es un texto y un conjunto de tareas de la misma. Los textos contienen información sobre eventos importantes, tradiciones, hazañas pasadas y modernas, y son materiales impresos o videos tomados de fuentes abiertas en Internet. Damos un ejemplo de BI, que incluye un video tomado de Internet, dedicado al Día de la Victoria con las tareas correspondientes.

Al desarrollar un conjunto de tareas, nos basamos en los trabajos de los investigadores, que en el curso del trabajo con el texto introducen una etapa de análisis de valores (Díaz, et al., 2017), precedida por las etapas de estudio del texto, evaluación de la situación y determinación de su propia posición, intercambio de opiniones, discusión grupal (Khramova, 2008, p. 9-10).

Creemos que para organizar eficazmente el proceso de creación de valores, el texto debe ir acompañado de tareas. El trabajo experimental ha demostrado que las tareas prácticas requieren conciencia, análisis e interpretación de los hechos, y en el futuro implican la transformación en VP de los estudiantes.

Para eliminar las dificultades léxicas antes del texto, es necesario realizar tareas léxicas y gramaticales, por ejemplo, para relacionar palabras y frases con su interpretación:

1. símbolo; 2. conmemorar ; 3. tradición; 4. fiesta; 5. exterminar.

a) destruir completamente;

b) objeto que se considera representativo de una entidad o de una idea;

c) doctrina, costumbre, etc., conservada en un pueblo por transmisión de padres a hijos;

d) recordar y mostrar respeto por alguien o algo;

e) jornada en que se celebra algo o que se dedica a alguien o algo.

Para actualizar los valores a nivel cognitivo, después de completar las tareas léxico-gramaticales, los estudiantes pasan al estudio del texto con una descripción y evaluación de la situación, por ejemplo: Las cintas de San Jorge son el símbolo más reconocido del recuerdo en Rusia y están estrechamente ligadas con el Día de la Victoria. ¿Cuáles son los símbolos del Día de la Victoria en Rusia? ¿Cómo se celebra el Día de la Victoria?

Con el fin de revelar la importancia de los problemas patrióticos, los estudiantes trabajan en pequeños grupos, expresan su propia posición, hay un intercambio de opiniones sobre una serie de preguntas, por ejemplo: ¿Es el Día de la Victoria una fiesta nacional en Rusia?

Este tipo de tareas implica la extracción de información del texto y su uso en un monológico, diálogo (razonamiento de su propia opinión) o una declaración polilógica (comparación y discusión de diversos aspectos).

Para organizar actividades reflexivas e involucrar a los estudiantes en el intercambio de opiniones, se organizan discusiones grupales bajo la dirección del maestro, por ejemplo: Exprese su opinión en 4-5 frases. Hay una tradición en Rusia para celebrar el Día de la Victoria con un desfile. Al mismo tiempo, los textos y las tareas son importantes para los estudiantes, lo que implica la inclusión de cada uno en el trabajo en un ejercicio en particular.

En la etapa final del análisis de valores, durante la etapa formativa del experimento, las tareas tienen como objetivo comprender y comparar los valores, por ejemplo: No se debe olvidar el pasado. ¿Qué significa para Ud. el Día de la Victoria? Debido a esto, apelando a las emociones de los estudiantes se organiza la actividad reflexiva y evaluativa. 


\section{Conclusiones}

Por lo tanto, se ha identificado que la formación de VP es un problema urgente en el campo de la enseñanza de la LE. La disciplina "Lengua extranjera" tiene un potencial formativo y patriótico.

El artículo presenta un análisis de los aspectos prácticos de la formación de PC utilizando el enfoque patriótico del valor MD en las clases de LE. El trabajo experimental realizado mostró que un medio eficaz para formar un VP es un conjunto de $\mathrm{MD}$, que incluye $\mathrm{BI}$, con un conjunto de tareas que contienen información sobre hazañas, grandes eventos históricos importantes para el país.

BI tienen una sola estructura: el texto y el complejo de tareas a la misma. Primero, los estudiantes realizan tareas para eliminar las dificultades léxicas y gramaticales, después de lo cual pasan al estudio del texto, la evaluación de la situación. Después de actualizar los valores a nivel cognitivo, los estudiantes deben determinar su propia posición durante una discusión grupal. Las tareas tienen como objetivo apelar a las emociones y luego a la reflexión y el análisis comparativo de los valores. Los alumnos participan en la actividad reflexiva y evaluativa, lo que les permite reflexionar sobre la procedencia de los VP.

Al mismo tiempo, las tareas tienen en cuenta la experiencia subjetiva de los estudiantes y los estimulan a expresarse sobre la base de problemas relevantes para la actualidad. Los materiales presentados pueden ser utilizados por los educadores en las clases de LE, así como por los investigadores del problema de la formación de valores.

\section{Referencias bibliográficas}

Adaeva, N. V. (2014). Educación patriótica de los estudiantes universitarios por medio de la pedagogía popular. (Tesis de Doctorado). Universidad Estatal de Oriol, Tver, Rusia.

Bespalko, V. P. (1977). Fundamentos de la teoría de sistemas pedagógicos: Problemas y métodos de apoyo psicológico y pedagógico de los sistemas de formación técnica. Vorónezh: Editorial de la Universidad Estatal de Vorónezh.

Bimakhanov, T. D., Ibragimov, G. I., Bulatbaeva, K. N., Murzabekov, M. K. y Kuatbekov, S. N. (2018). Tradiciones del desarrollo físico en la pedagogía nacional kazaja como medio de educación de futuros maestros. Espacios. vol. 39(49), p. $17 . \quad$ Recuperado de: http://www.revistaespacios.com/a18v39n49/a18v39n49p17.html

Blauberg, I. V. (1997). Problema de integridad y enfoque sistemático. Moscú: Editorial URSS.

Burylova, L. A. (2004). Metodología para la creación y uso de materiales didácticos sobre lecciones de física para estudiantes del 5-7 grados. (Tesis de Doctorado). Universidad Estatal Pedagógica, Cheliabinsk, Rusia.

Derkach, A. A. (2004). Acmeología. Moscú: RAGS.

Díaz, L. C., Rondón, M., Sánchez, L. C., Camejo, L. M., y Espinosa, E. M. (2017). Actividades Artísticas, una vía para fortalecer el valor patriotismo en los estudiantes de las Ciencias Médicas. Multimed. 19(1), pp. 201-214.

Filashova, O. A. (2015). Educación patriótica por medio de un idioma extranjero en la escuela primaria. Experimento e innovación en la escuela, vol. 2, pp. 14-16.

Gashchenko, L. A. (2004). La ideología patriótica y sus componentes. Revista de Academia Estatal de Vitebsk, vol. 2(32), pp. 9-14. 
Khramova, E. B. (2008). La formación de especialistas en el proceso de aprendizaje de una lengua extranjera en una universidad técnica. (Tesis de Doctorado). Universidad Estatal de Ryazan, Ryazan, Rusia.

Kiryakova, A. V. (1996). Teoría de la orientación de la personalidad en el mundo de los valores. Orenburg, Rusia.

Konysheva, A. V. (2017). A la cuestión de la educación patriótica en la escuela superior por medio de la lengua extranjera. Revista de GGU. Recuperado de: http://Ip009.flfm.ru/vestnik_ggu

Koroleva, S. S. (2010). Formación de los valores de los estudiantes en las universidades agrícolas en el proceso de enseñanza de una lengua extranjera. (Tesis de Doctorado). Universidad Estatal Pedagógica, Kaluga, Rusia.

Novikov, A. M. (2013). Pedagogía: un diccionario de un sistema conceptual básico. Moscú: IET Centro de Publicaciones.

Orlov, V. B., Lesnyak, V. I. (2006). El patriotismo como valor de la sociedad civil. Revista de Universidad Estatal de Ugra, vol. 3, pp. 80-86.

Rogova, G. V., Vereshchagina, I. N. (2000). Métodos de enseñanza de inglés. Moscú: Prosvescheniye.

Rubinstein, S. L. (2003). Ser y conciencia. El hombre y el mundo. San Petersburgo: Piter.

Rusetskiy, A. V., Gashchenko, L. A. (2000). Los valores patrióticos de los estudiantes de Vitebsk al comienzo del nuevo milenio. Revista de Academia Estatal de Vitebsk, vol. 1 (15), pp. 3-15.

Rusia muestra novedades militares en el desfile del Día de la Victoria (2018). Recuperado de: https://es.euronews.com/embed/465045

Salikov, D. Kh. (2011). El desarrollo de los valores patrióticos de los estudiantes en el proceso de formación en la universidad. (Tesis de Doctorado). Universidad estatal de los Urales del sur, Cheliabinsk, Rusia.

Serikov, V. V. (1998). La educación orientada personalmente: la búsqueda de un nuevo paradigma. Moscú: Editorial UEPV.

Shaidullin, T. V., Larina, T. V. (2016). Educación patriótica del personal militar del Ejército Popular de Liberación de China. Foro científico. Recuperado de: https://scienceforum.ru/2016/article/2016022022

Slastenin, V. A. (2003). Introducción a la axiología pedagógica. El manual para estudiantes de universidades pedagógicas. Moscú: Editorial Academia.

Sukhanova A. S. (2020). Niveles de formación de los valores patrióticos en el proceso educativo de la Universidad. Pedagogía. Cuestiones de teoría y práctica, 6, pp. 734-737. DOI: 10.30853/ped200152

Vishnevsky, E. I. (1988). Posibilidades educativas del proceso de enseñanza de lenguas extranjeras. Idiomas extranjeros en la escuela, vol. 4, pp. 23-28.

Vyrschikov, A. N., Kusmartsev, M. B. (2006). Educación patriótica de la juventud en la sociedad rusa moderna. Volgograd: NP IPD "Avtorskoye Pero".

Yakimanskaya, I. S. (1995). La tecnología de aprendizaje orientado a la personalidad. Problemas de Psicología, vol. 2, pp. 31-42.

Zeer, E. F. (2006). Psicología de las profesiones: manual para estudiantes universitarios. Moscú: Proyecto académico; Fundación "Mir".

Esta obra está bajo una Licencia Creative Commons

Attribución-NoCommercial 4.0 International

(c) EY-NO 